\title{
Numerical Simulation for Crushing of Shell Material about 50SiMnVB Influenced by Brittle Zone
}

\author{
Ren-yi Wan* and De-yuan Li \\ School of Materials Science and Engineering, Shenyang University of Technology, \\ Shenyang 110870,Liaoning,China
}

Keywords: 50SiMnVB; Explosive Fragmentation; Brittle Fracture.

\begin{abstract}
We use the finite element software ANSYS to verify the explosion experiment about whether the projectile shell contains brittle zone, and the brittle with different width and depth to improve the fragmentation rate and lethality of shell material about 50SiMnVB. The simulation results show that the shell with a brittle zone is more explosive than with out's; Within the allowable range, the bigger width of the brittle zone, the more uniform broken shell, the greater the projectile damage; the deeper depth of the brittle zone, the more easily the shell be broken, and fragment size close, the greater the projectile damage.
\end{abstract}

\section{Introduction}

In military industry, the broken degree and fragmentation rate of shell is the important standard to judge the projectile lethality. In order to improve the projectile lethality, our shell material changed with advances in technology. The 50SiMnVB is the most widely used now days. Without quenching the strength of 50SiMnVB can reach more than four hundred megapascals, so that the effective fragmentation rate has been greatly improved[1-4]. Under the condition of stress, brittle materials will produce brittle fracture which are more easily fracture compared with ductile materials in the same force. In order to improve the fragmentation of projectile shell and fragment mass destruction, using laser to machine a brittle zone on the shell with 50SiMnVB to research the effect of brittle zone to the shell material crushing process under the explosive impact[5-6]. Considering the limiting of actual validation criteria, the numerical simulation software ANSYS / LS-DYNA is selected to simulate and analyze the process of the particular experiment.

\section{Modeling}

Explosion occurs at high temperature, high pressure, and high strain rates. ANSYS software is based on energy, momentum, mass conservation equations of projectile explosion simulation study[7-9]. Simulation model was a quarter of the projectile, as shown in figure.1. Specific dimensions were explosives outside diameter $112 \mathrm{~mm}$, projectile shell inner diameter $112 \mathrm{~mm}$ and outside diameter $122 \mathrm{~mm}$, the projectile height $60 \mathrm{~mm}$. High explosive material models and JWL equation of state were chosen as explosive material model. Plastic kinematic hardening model PLASTIC_KINEMATIC was chosen as the model of brittle materials which could be a good description of brittle fracture process. JOHNSON COOK model which is very suitable for the study material crushing process in the situation of high temperature, high pressure, and high strain rate and GRUNEISEN equation of state is chosen as projectile shell material. GRUNEISEN equation of state material pressure is defined as follows:

$$
p=\frac{\rho_{0} C^{2} \mu\left[1+\left(1-\frac{\gamma_{0}}{2}\right) \mu-\frac{a}{2} \mu^{2}\right]}{\left[1-\left(S_{1}-1\right) \mu-S_{2} \frac{\mu^{2}}{(\mu+1)}-S_{3} \frac{\mu^{3}}{(\mu+1)^{2}}\right]^{2}}+\left(\gamma_{0}+a \mu\right) E
$$


$\mathrm{C}, \mathrm{S} 1, \mathrm{~S} 2, \mathrm{~S} 3, \gamma 0$, a are required input parameter[10]. In this article, $\mathrm{C}=0.457, \mathrm{~S} 1=1.49, \gamma 0=2.17$, $\mathrm{a}=0.46$, the remaining input parameters are 0 .

The contact between explosives and shell use CONTACT_SLIDING_ON-LY_PENALTY things to slip contact algorithm in the model. Other parts use the basic CONTACT SURFACE TO SURFACE contacts. Because of a quarter of the projectile shell, adding constraints on the left and right sides of the model, casing and brittle surface. Using mm-g- $\mu$ s modeling, computational time is $200 \mu \mathrm{s}$, and the average output simulation results per $1 \mu \mathrm{s}$.

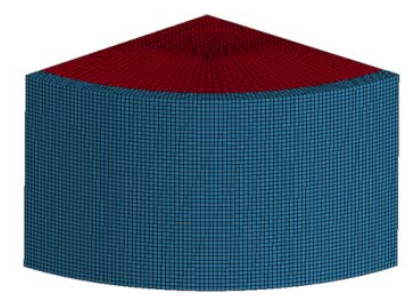

Fig.1. Calculation model of projectile shell

\section{Simulation and Analysis}

In order to analyze whether brittle zone has the significant influence on the material crushing, the model with brittle zone and no brittle zone were established firstly Then analyze the influence of the depth and width of a brittle zone to the material crushing. The article established four groups models with different shades and width to simulate compare within the machining parameters allowable range. The results are as follows:

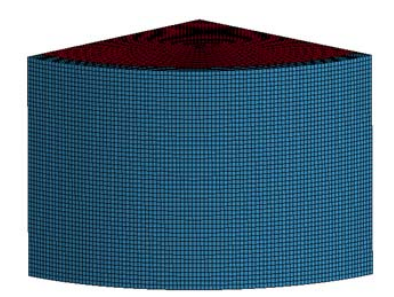

(a) model no brittle zone

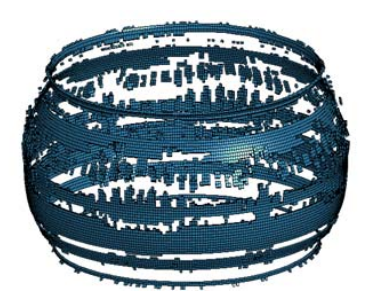

(b) crushing state no brittle

Fig.2. Simulation diagram of no brittle projectile shell

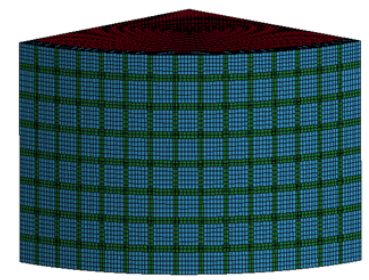

(a) model with brittle zone

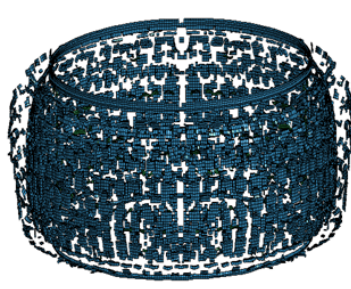

(b) crushing state with brittle zone

Fig.3. Simulation diagram of projectile shell with brittle

\section{Influence of Brittle Zone to the Model Crushing Processing}

Projectile with 50SiMnVB shell material which with brittle zone and without brittle zone models are shown as figure. 2 and the crushing state after $30 \mu$ s is shown as figure.3. Simulation period is $200 \mu \mathrm{s}$, and each $1 \mu \mathrm{s}$ outputs a result data file. According to the figure.2, we can see clearly that at the same time, the degree of projectile fragmentation with a brittle zone is significantly greater than the 
extent of the shell with no brittle. The part which cracked extend with brittle zone. With the uniform fragments size, the fragments rate with brittle zone are greater than without brittle.

\section{Influence of the Brittle Zone Depth to the Model Crushing}

Different depths of the brittle zone have different effects to the model crushing. Four groups model with depth $0.7 \mathrm{~mm}, 1.3 \mathrm{~mm}, 2.3 \mathrm{~mm}, 4 \mathrm{~mm}$ and width $3 \mathrm{~mm}$ determined by processing parameters. The crushing state at $30 \mu \mathrm{s}$ is shown in figure.4. The simulation results shown that at the same time, the same brittle width, the bigger depth the more uniform the shell crushing pieces. The shorter time of crushing completely, the greater the degree of fragmentation scattering. These declared that the depth of brittle zone has a significant influence on the projectile shell crushing. Within a reasonable range of processing parameters, the deeper brittle, the better the impact of the projectile fragmentation.

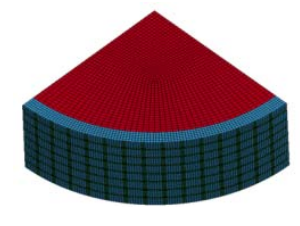

(a) $0.7 \mathrm{~mm}$

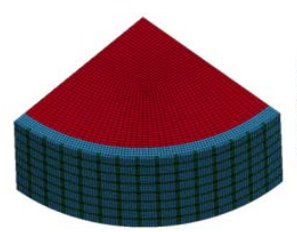

(c) $2.3 \mathrm{~mm}$
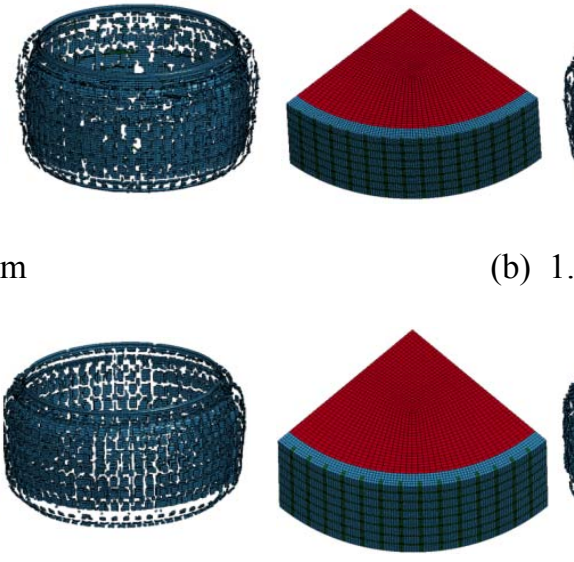

(d) $4 \mathrm{~mm}$

Fig.4. Simulation diagrams of projectile shell and crushing state with different depth brittles

\section{Influence of the Brittle Zone Depth to the Model Crushing}

Four groups model with width $0.9 \mathrm{~mm}, 1.6 \mathrm{~mm}, 3 \mathrm{~mm}, 5 \mathrm{~mm}$ and depth $4 \mathrm{~mm}$ determined by processing parameters. The crushing state at $30 \mu \mathrm{s}$ is shown in figure 5. The simulation results showed that at the same time, the same brittle depth, the bigger width the more uniform the shell crushing pieces. The shorter time of crushing completely, the greater the degree of fragmentation scattering. These declared that the width of brittle zone has a significant influence on the projectile shell crushing. Within a reasonable range of processing parameters, the wider brittle, the better the impact of the projectile fragmentation.

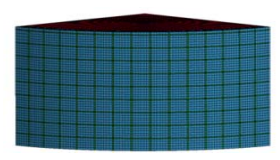

(a) $0.9 \mathrm{~mm}$
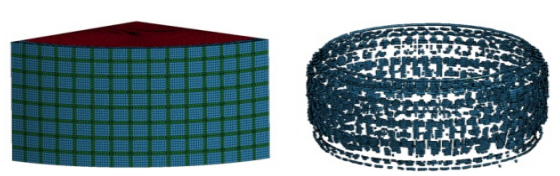

(c) $1.6 \mathrm{~mm}$

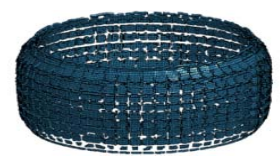

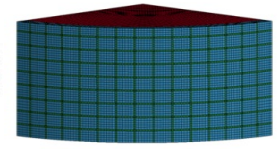

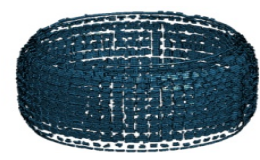

(b) $1.6 \mathrm{~mm}$
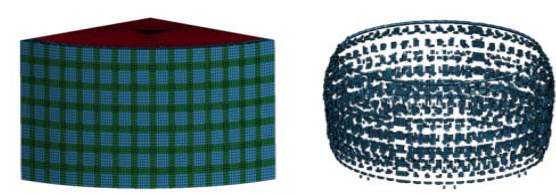

(d) $5 \mathrm{~mm}$

Fig.5. Simulation diagrams of projectile shell and crushing state with different width brittles 


\section{Stress Map Changes Between Brittle Zone and No Brittle}

The stress state of projectile with brittle and no brittle was shown in figure 6 . We can see clearly that the stress distribution was very uneven when there was no brittle in the crushing process and no law. The crushing lasted longer, fragment sizes, uneven distribution, and irregular fracture in the rupture boundary of the shell. The stress concentrated in brittle zone when the projectile existed brittle zone, and uniform distribution, crushing quickly, scattering large fragments with same size. The boundary of the shell with brittle zone was more regular than the no brittle.

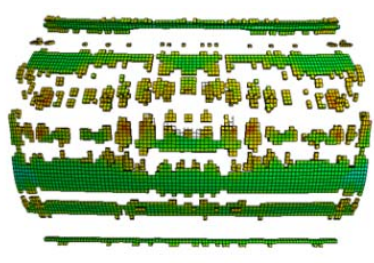

(a) No brittle zone

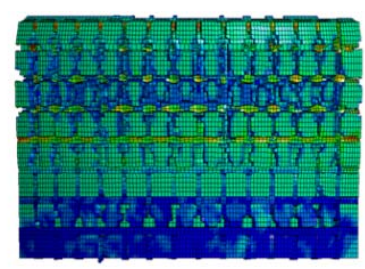

(b) with Brittle zone

Fig.6. Stress distribution diagram of broken model

\section{Internal Power of Different Parameters with Brittle Zone}

Brittle zone of different width and depth had a certain degree of influence on the broken projectile shell, which shown in figure 7 and 8 . The larger width of brittle zone, the greater internal energy, the more easily reach the yield limit, the greater the degree of stress concentration brittle zone area, the more obvious crushing degree, the deeper brittle zone, the greater internal energy. The more easily achieve to fracture yield, the greater degree of stress concentration in the brittle zone area and the more easily broken.

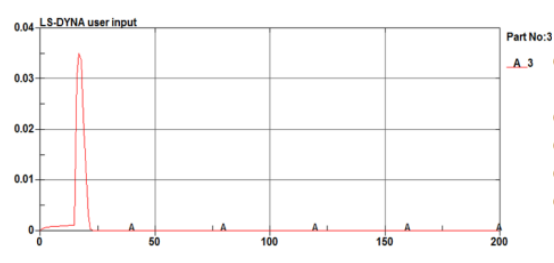

(a) Internal energy curves of small width brittles

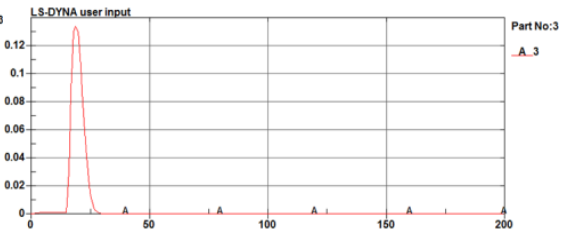

(b) Internal energy curves of large width brittles

Fig.7. Internal energy curves of different width brittles

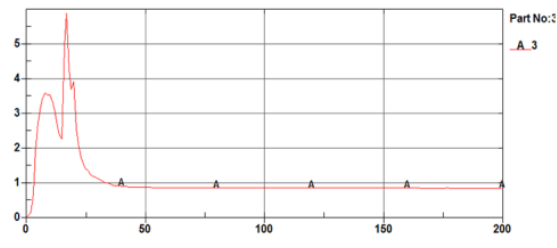

(a) Internal energy curves of small depth brittles

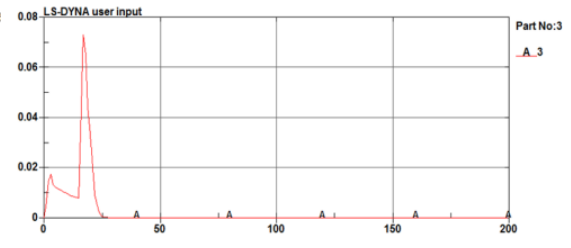

(b) Internal energy curves of large depth brittles

Fig.8. Internal energy curves of different depth brittles

\section{Conclusions}

Using the finite element software ANSYS to simulate the projectile fragmentation process of 50SiMnVB. The simulations contained the study of different brittles zone, width and depth to the 
broken state diagram, stress state diagram and the internal energy of brittle zone effect. The conclusions are following:

1.Because of the brittles zone which produces stress concentration, when the projectile with 50SiMnVB blasting, more shell rupture along with brittle zone, the greater degree of crushing, fragments of relatively uniform size, and lethality larger than no brittle.

2.In the allowable range of actual situation, different width brittles have different projectile crushing. From the simulation results, wider brittle zone, more severe stress concentration, greater international energy, more easily broken brittle zone area, greater degree of shell crushing, more evenly fragment distributed and more similar size fragments.

3.In the allowable range of actual situation, different depth brittles have different projectile crushing. From the simulation results, deeper brittle zone, more severe stress concentration, greater international energy, more easily broken brittle zone area, greater degree of shell crushing, more evenly fragment distributed and more similar size fragments.

\section{References}

[1] Li Z H, Shi J P, Tang A M. Journal of Xi'an University of Technology, 2011, 27(3): 280-284.

[2] Li Z H, Shi J P, Tang A M. Journal of Applied Mechanics, 2012, 29(1) : 48-50.

[3] Jiang Y C, Xu S W, Chen H. Engineering Mechanics, 2008, 25(4): 50-54.

[4] Chen Q X. Inner Mongolia Petrochemical Industry, 2014(14): 21-22.

[5] Li D Y, Zhao L, Wan R Y, et al. Journal of Shenyang University of Technology, 2014, 36(2): 154-158.

[6] Li D Y, Wang Y, Wan R Y. Journal of Shenyang University of Technology, 2015, 37(3): 278-282.

[7] Li J F. Journal of Nanjing University of Technology, 2006, 30(2) : 186-188.

[8] Lan Y L, Wan R Y. Equipment Manufacturing Technology, 2013(12): 177-178.

[9] Shang X J, Su J Y, et al. ANSYS / LS-DYNA dynamic analysis methods and project examples, China Water Power ,Beijing, 2005. 